\title{
SPETTROSCOPIA MUONICA: UNA SONDA MICROSCOPICA PER IL MAGNETISMO
}

\author{
SAMUELE SANNA (*) \\ Nota presentata dal m.e. Attilio Rigamonti \\ (Adunanza del 23 giugno 2011)
}

SUNTO. - La spettroscopia muonica è una tecnica sperimentale che impiega fasci di muoni per studiare le proprietà della materia condensata. In particolare essa permette lo studio di processi statici e dinamici in materiali magnetici di varia natura (ferro magneti/antiferromagneti, vetri di spin, frustrati, etc) e superconduttori, come anche di reazioni chimiche e fenomeni di diffusione di particelle cariche e neutre.

In queste pagine verranno descritti a livello elementare i principi fisici che stanno alla base di questa tecnica, evidenziandone soprattutto l'impiego nello studio microscopico delle proprietà magnetiche dei materiali.

$* * *$

ABSTRACT. - The muon spectroscopy is an experimental technique which employs muon beams to study the properties of condensed matter. In particular, it allows us to study static and dynamic processes in magnetic materials (e.g. ferromagnets/antiferromagnets, spin glasses, frustrated, etc) and superconductors, as well as chemical reactions and diffusion phenomena of charged and neutral particles.

Here we will describe at the elementary level the physical principles of this technique, highlighting especially the use in the microscopic study of the magnetic properties of materials.

(*) Dipartimento di Fisica, via Bassi 6, Università di Pavia.

E-mail: samuele.sanna@unipv.it 


\section{INTRODUZIONE}

Fin dall'antichità l'uomo si è chiesto come si comportasse la materia a livello microscopico. Nel corso del '900 i notevoli progressi della ricerca in Fisica hanno reso possibile la scoperta e la classificazione delle particelle elementari, cioè i costituenti indivisibili della materia. Essi sono i quark e i leptoni, noti come particelle-materia, ai quali si aggiungono i mediatori delle forze esistenti in natura, $\mathrm{i}$ bosoni di gauge, come per esempio il fotone. I quark sono i "mattoni" che formano protoni e neutroni, mentre l'elettrone è un leptone e insieme formano gli atomi, cioè la materia che ci circonda e di cui noi stessi siamo fatti. Ad essi si aggiungono altre particelle più esotiche, come leptoni meno noti o combinazioni di quark, che hanno tempi di vita piuttosto brevi. Un leptone che risulta particolarmente rilevante per studiare le proprietà microscopiche della materia è il muone. In ogni minuto il nostro corpo viene colpito da decine di muoni trasportati dai raggi cosmici che attraversano l'atmosfera.

Questi purtroppo giungono con tempi e direzioni casuali quindi non adatti per realizzare esperimenti. A questo scopo è necessario produrre intensi fasci di muoni in laboratorio con flusso, direzione e velocità controllati. In tal modo i muoni possono essere utilizzati come microscopiche bussole in grado di interagire con le proprietà magnetiche della materia condensata. Ciò è possibile in virtù del fatto che il muone possiede momento magnetico intrinseco o spin $1 / 2$ (come l'elettrone) e poco dopo aver interagito con la materia decade, essendo un leptone instabile, trasformandosi in altre particelle elementari tra cui il positrone. Quest'ultimo è il messaggero che contiene la preziosa informazione cercata: la direzione in cui viene deviato l'ago della bussola microscopica, cioè lo spin, dall'interazione con la materia. Con un eccesso di immaginazione, possiamo pensare il muone come un esploratore introdotto in un mondo sconosciuto da cui non può fare ritorno, ma che nel morire lancia un sassolino che individua la direzione in cui punta l'ago della sua bussola. Tutto avviene obbedendo rigorosamente alle leggi specifiche del mondo microscopico, cioè quelle della meccanica quantistica.

Nella spettroscopia muonica si misura al variare del tempo l'orientazione di un gran numero di questi speciali esploratori "quantistici” da cui è possibile ricostruire le proprietà magnetiche microscopiche del materiale studiato. 


\section{PRODUZIONE DI MUONI}

In natura esistono muoni di carica sia negativa che positiva. I primi $\left(\mu^{-}\right)$sono poco efficaci per studiare la materia, in quanto facilmente collassano nel nucleo atomico (di carica positiva) da cui sono attratti, e l'effetto è un po' come perdere la bussola. I secondi $\left(\mu^{+}\right)$invece vengono attratti dalle nuvole elettroniche e diventano diretti spettatori della fisica degli elettroni, che sono i veri protagonisti delle proprietà della materia condensata.

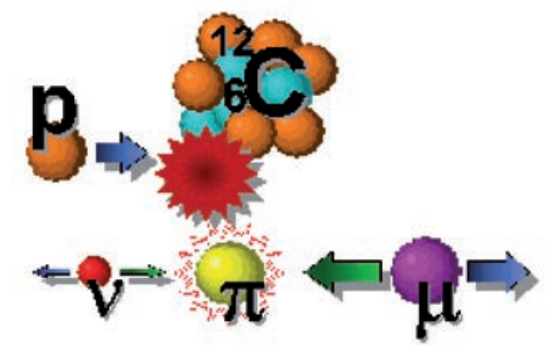

Fig. 1 - Processo schematico per la produzione di muoni.

In laboratorio i $\mu^{+}$si ottengono inviando fasci di protoni ad alta energia cinetica su un bersaglio (solitamente di grafite). Per far ciò si devono utilizzare speciali acceleratori di particelle, sincrotroni o ciclotroni, in grado di conferire loro energie sufficienti (almeno $500 \mathrm{MeV}$ ) affinchè l'urto col bersaglio possa produrre i pioni positivi, $\pi^{+}$, che a loro volta dopo un tempo di vita brevissimo ( 20 miliardesimi di secondo) decadono producendo ciascuno un $\mu^{+}$e un neutrino, $v_{\mu}$,:

$$
\pi^{+} \rightarrow v_{\mu}+\mu^{+}
$$

I neutrini difficilmente si riescono ad acchiappare, mentre $\mathrm{i}$ muoni possono essere raccolti e inviati all'interno di un materiale. L'intero processo è schematizzato in Fig. 1.

Un impianto per la produzione di muoni essenzialmente è composto di un sincrotrone dove vengono prodotti e accelerati i protoni, un bersaglio di grafite, opportune linee di raccolta e trasporto dei muoni e gli spettrometri per la realizzazione degli esperimenti. Per poter realizzare un esperimento di spettroscopia muonica è necessario disporre di un fascio di muoni con direzione iniziale degli spin nota e uguale per tutti. Ciò è possibile sfruttando una caratteristica specifica del neutrino, $v_{\mu}$, prodotto 
nel decadimento del pione $\pi^{+}$: il suo spin ha sempre direzione opposta rispetto alla direzione in cui si muove (dicesi che ha elicità negativa).

Per aiutarci a cogliere il significato di questo fenomeno possiamo partire da un esempio a tutti familiare, ispirato dal mondo macroscopico: il fatto che un gatto cada sempre in piedi. Infatti un gatto che per caso si trovi in volo col dorso rivolto verso il basso ruota la coda per rigirarsi (Fig. 2).
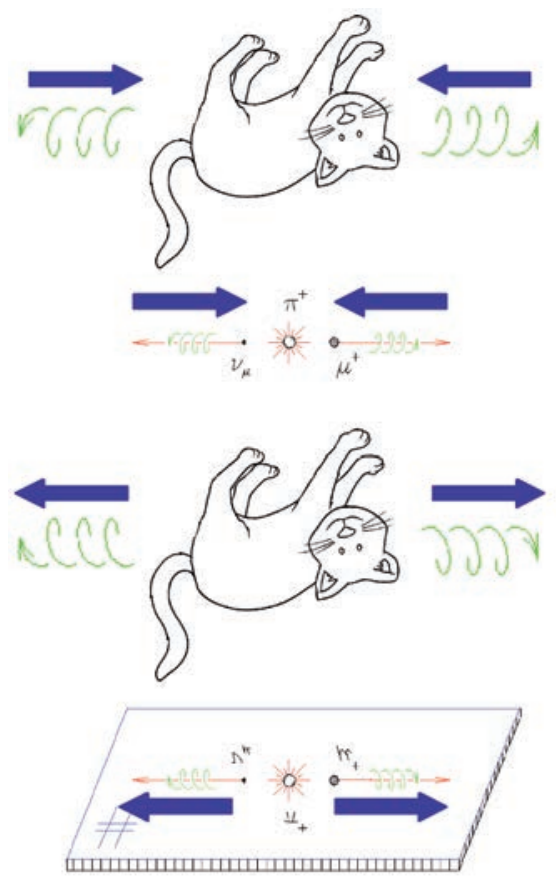

Fig. 2 - Rappresentazione schematica della violazione parità. Nel decadimento del muone il processo rappresentato nel pannello inferiore è proibito.

Così facendo produce un momento angolare, una quantità fisica che possiamo esprimere con un vettore il cui verso indica se la coda ruota in senso orario o antiorario. Come reazione il resto del corpo ruota in senso opposto, in virtù del principio di conservazione del momento angolare. Sempre in un eccesso di immaginazione, associamo il neutrino alla coda e il muone al corpo del gatto e immaginiamoli come due sferette che ruotano attorno al proprio asse. Il loro spin è direttamente legato al momento angolare intrinseco dovuto a questa rotazione. La coda resta 
attaccata al gatto, per sua fortuna, invece neutrino e muone vengono sparati in direzioni opposte tra loro. Ma vi è una differenza ancora più importante: il gatto può girare la coda indifferentemente in senso orario o antiorario, puntando il momento angolare (la freccia nel disegno) associato a questo movimento a destra o sinistra. Invece neutrino e muone hanno elicità ben definita, cioè hanno sempre spin orientato nel verso opposto rispetto alla direzione in cui si propagano. Il viceversa in natura non è permesso. Questa violazione della simmetria, nota come violazione della parità, si verifica solo nel mondo microscopico e in modo particolare riguarda il decadimento dei leptoni.

Pertanto i muoni, se prodotti da pioni immobili sulla superficie del bersaglio di grafite, si allontanano tutti alla stessa velocità puntano il loro spin all'indietro. In questo modo vengono realizzati fasci di muoni spinpolarizzati al $100 \%$ in una direzione iniziale ben nota e facilmente, attraverso l'impiego di campi elettrici e magnetici, si possono manipolare ed inviare all'interno del materiale che si desidera studiare.

\section{IL MUONE POSITIVO DENTRO IL MATERIALE: UNA BUSSOLA QUANTISTICA}

La possibilità di impiantare muoni positivi dentro la materia è principalmente legata a due proprietà: 1) la sua massa circa 10 volte più piccola di quella del protone e 2) la sua carica elettrica positiva. Infatti, sebbene appartenga alla famiglia dei leptoni come l'elettrone, ai nostri scopi possiamo immaginare il muone positivo come un protone di dimensioni ridotte. In questo modo riesce a penetrare la materia e, interagendo con le cariche elettriche circostanti, a termalizzare molto rapidamente, cioè perde la sua energia cinetica (di circa $4 \mathrm{MeV}$ ) in meno di un miliardesimo di secondo. Durante il processo di termalizzazione il muone ha comunque modo di penetrare per qualche centinaio di micron, a seconda della densità del materiale (per esempio nell'acqua ha uno stopping range di $140 \mathrm{mg} / \mathrm{cm}^{2}$, cioè si ferma dopo $1.4 \mathrm{~mm}$ ), quindi sulla scala atomica ben oltre la superficie del materiale. Poichè le interazioni frenanti sono di natura Coulombiana, in questa fase l'orientazione del suo spin resta inalterata, requisito fondamentale per il successo della spettroscopia muonica. Il muone positivo in alcuni casi forma uno stato legato muoneelettrone, il muonio, il cui comportamento non verrà qui trattato. Consideriamo quindi il muone nel suo stato libero in cui una volta ter- 
malizzato nella materia assiste come "spettatore" dei processi elettronici, specialmente quelli legati al magnetismo.

La capacità di sondare lo stato magnetico di un materiale risiede nel fatto che il muone possiede uno spin $1 / 2$, immaginabile come un aghetto magnetico microscopico. In virtù di esso si instaura una interazione di natura dipolare tra il muone e i campi magnetici localmente prodotti per esempio da momenti magnetici, correnti microscopiche o spin. Infatti in presenza di un campo magnetico locale $B_{l o c}$ lo spin, anzichè orientarsi lungo il campo come farebbe l'ago di una bussola, compie un moto di precessione con una frequenza angolare di Larmor $\omega_{\mathrm{L}}=\gamma_{\mu} B_{\text {loc }}$ (dove $\gamma_{\mu}=$ $2 \pi \bullet 136 \mathrm{MHz} / \mathrm{T}$ è il rapporto giromagnetico del muone) attorno alla direzione del campo. Un comportamento analogo si ritrova anche nel mondo macroscopico osservando il moto di precessione di una trottola attorno alla direzione del campo gravitazionale terrestre (Fig. 3).
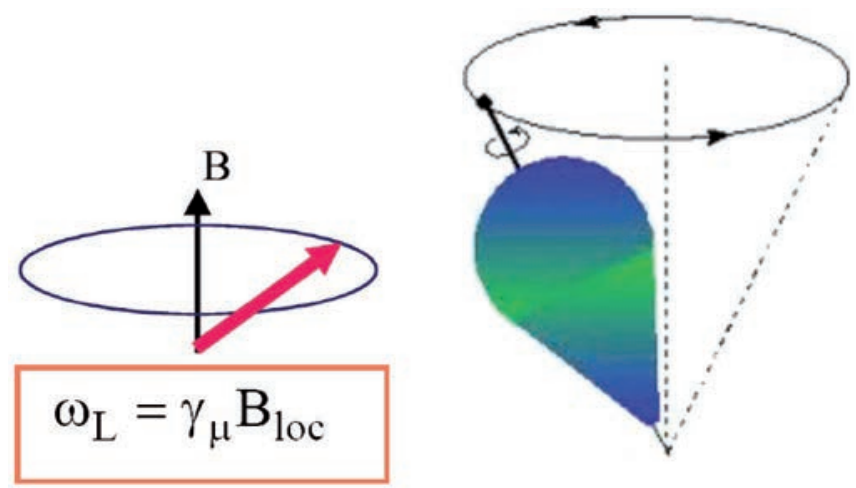

Fig. 3 - Il moto di precessione dello spin del muone attorno al campo magnetico Bloc nel mondo macroscopico trova analogia meccanica col moto di precessione della trottola attorno al campo gravitazionale terrestre.

Il muone è una particella instabile che decade mediamente in un tempo di $2.2 \mu$ s, piuttosto lungo rispetto al tempo caratteristico dei processi elettronici. Pertanto si comporta come una sonda di processi che possiamo definire statici o quasi-statici, come per esempio l'eventuale ordinamento di momenti magnetici presenti nel materiale.

Come fa il muone a fornirci informazioni su ciò che osserva? La ragione per cui un muone è in grado di fornirci informazioni sulla natura del materiale studiato è che esso decade emettendo un positrone (un 
elettrone con carca positiva $e^{+}$) preferenzialmente lungo la direzione del suo spin. Infatti il decadimento del muone produce anche due neutrini e, analogamente a quanto osservato nel decadimento del pione descritto in precedenza, per la violazione del principio di conservazione della parità, la distribuzione spaziale dei positroni emessi è asimmetrica.

In questo fenomeno si può sfruttare il positrone come messaggero dell'informazione cercata, rilevando la direzione in cui il positrone viene emesso per mezzo di opportuni scintillatori a stato solido che vengono distribuiti attorno al materiale da misurare.

\section{L'ESPERIMENTO DI SPETTROSCOPIA MUONICA}

In un tipico esperimento base di spettroscopia muonica si posizionano due rivelatori, uno posto anteriormente e uno posteriormente il materiale rispetto alla direzione del fascio di muoni, e si misura la differenza del numero di positroni emessi tra le due direzioni (Fig. 4). Se con $\mathrm{N}_{\mathrm{A}}$ e $\mathrm{N}_{\mathrm{P}}$ si indicano i conteggi dei positroni misurati questo si può esprimere con la relazione:

$$
\frac{N_{A}-N_{P}}{N_{A}+N_{P}}=A(t)=a_{O} P(t)
$$

La somma a denominatore è utile per normalizzare rispetto al numero totale di muoni, che decadono con la legge esponenziale $N(t)=\mathrm{N}_{\mathrm{o}} \mathrm{e}^{\mathrm{t} / \tau}$ dove $\tau=2.2 \mu$ s è il tempo di vita medio. In questo modo al tempo iniziale $\mathrm{t}=0$ il rapporto deve essere uguale proprio all'asimmetria $a_{o}$ propria del decadimento del muone (teoricamente 0.33 ): $A(0)=a_{o}$ o equivaletemente $P(0)=1$. In funzione del tempo essa varia con una funzione che dipende dalle caratteristiche del materiale studiato. In pratica, per quanto detto sopra, è sensibile a tutti i fenomeni che producono una variazione dell'orientazione dello spin del muone. L'espressione (1) descrive quindi la distribuzione statistica temporale della polarizzazione dello spin di un numero elevato di muoni (tipicamente dell'ordine di 1 milione). Poichè l'orientazione dello spin del muone, e quindi l'asimmetria del suo decadimento, dipende dalla natura delle interazioni magnetiche con il materiale, l'asimmetria $\mathrm{A}(\mathrm{t})$ contiene informazioni sulle proprietà magnetiche microscopiche del materiale studiato. 


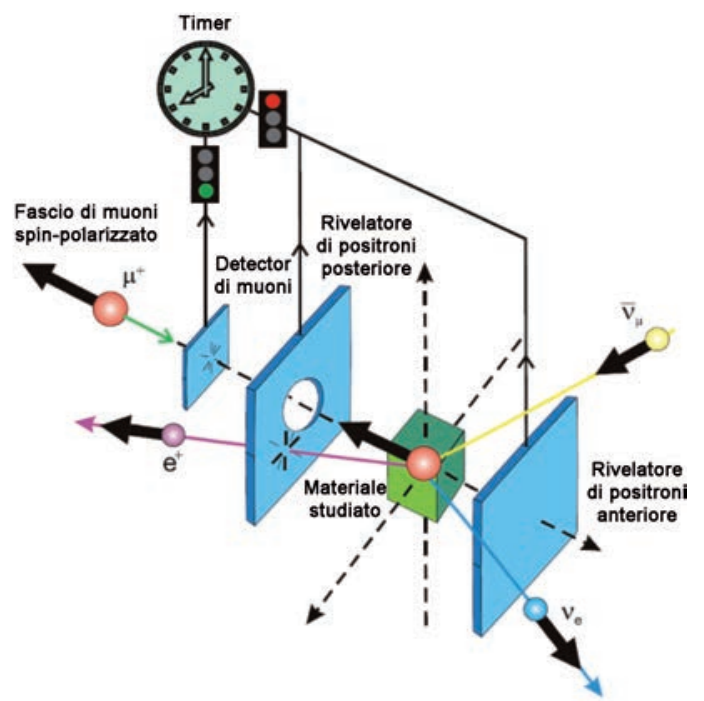

Fig. 4 - Schema di un tipico esperimento di spettroscopia muonica. Sulla sinistra, un fascio di muoni con spin polarizzati $100 \%$ all'indietro viene inviato nel campione dove, dopo aver interagito con i microscopici campi magnetici interni, decade emettendo due neutrini, difficilmente rilevabili, e un positrone che può essere intercettato dai due rivelatori. Poiché il positrone è preferenzialmente emesso lungo la direzione dello spin del muone, la cui orientazione è modificata dalle interazioni col materiale, è possibile studiare le proprietà magnetiche microscopiche del materiale dalla differenza del numero di muoni emessi anteriormente e posteriormente il campione.

Per esempio in un materiale paramagnetico, privo di ordinamento degli spin elettronici, la forma funzionale è molto semplice: $A(t)=$ $a_{0} G(t)$, in cui l'asimmetria diminuisce nel tempo con una semplice funzione di rilassamento di tipo gaussiano $G(t)=\exp \left(-\sigma^{2} t^{2}\right)$, principalmente dovuto all'interazione con deboli momenti magnetici disordinati (solitamente nucleari). Il processo si può immaginare come causato dal fatto che ciascun muone interagisce con un piccolo campo locale che fluttua casualmente perturbando l'orientazione iniziale del muone. La somma vettoriale dei contributi, scorrelati fra loro, degli spin di tutti i muoni pertanto tende lentamente a zero producendo la progressiva depolarizzazione del fascio iniziale, descritta appunto dalla funzione di rilassamento $G(t)$.

Se invece nel materiale si instaura un ordinamento magnetico a lungo raggio, per esempio ferromagnetico, tutti gli spin interagiscono con 
un campo magnetico locale $\mathrm{B}_{\text {loc }}$ in modo coerente. Questo processo produce la rotazione della polarizzazione di spin e quindi dell'asimmetria con una frequenza angolare pari alla frequenza di Larmor $\omega_{L}=\gamma_{\mu} B l o c$. Se per esempio il materiale è un monocristallo posto in modo tale che la polarizzazione iniziale sia perpendicolare alla direzione del campo interno Bloc, l'asimmetria del decadimento può essere descritta come:

$$
A(t)=a_{0} G(t) \cos \left(\omega_{L} t\right)
$$

in cui la frequenza angolare contiene, come già sappiamo, informazioni sul valore del campo interno statico Bloc, e la funzione di rilassamento $G(t)=\exp \left(-\sigma^{2} t^{2}\right)$ riflette la larghezza della distribuzione del valore del campo (il coefficiente $\sigma$ infatti risulta proporzionale al momento secondo della distribuzione) (Fig. 5). La depolarizzazione sarà più rapida in un sistema in cui l'intensità del campo locale varia significativamente al variare della posizione (per esempio a causa della presenza di impurezze, difetti o anche di qualche modulazione spaziale intrinseca come nel caso del reticolo magnetico dei superconduttori), in quanto ciascun muone precede ad una frequenza leggermente differente da quello posto in una posizione diversa, producendo la rapida decoerenza del segnale.

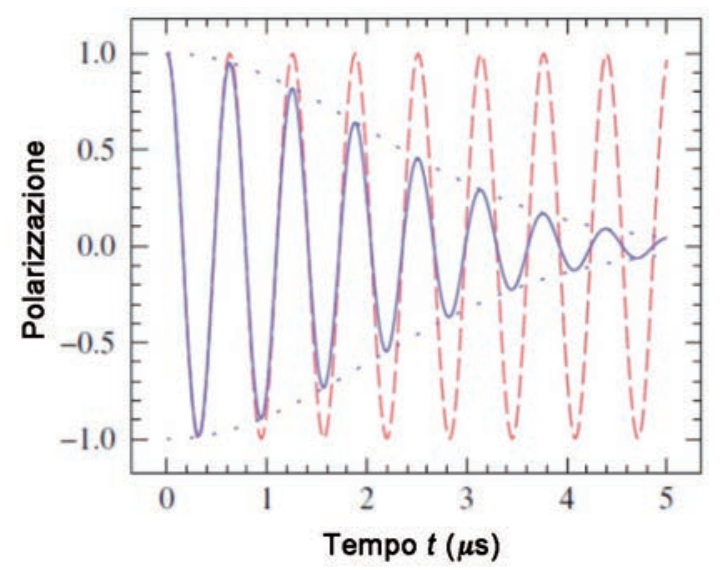

Fig. 5 - Esempio di evoluzione temporale della polarizzazione del muone in presenza di un campo magnetico Bloc perpendicolare alla polarizzazione iniziale.

La linea tratteggiata rappresenta il caso ideale in cui non sono presenti fenomeni $d i$ depolarizzazione, cioè $G(t)=1$. La linea solida si ha ponendo $G(t)=\exp \left(-\sigma^{2} t^{2}\right)$, tipico di una distribuzione statica di campi locali. 
Gli esperimenti vengono realizzati anche in presenza di campo esterno, in direzione sia perpendicolare che parallela alla polarizzazione iniziale. Quanto finora detto è già sufficiente per intuire che la spettroscopia muonica è adatta a studiare tutti i vari tipi di magnetismo che si osservano in natura principalmente attraverso la misura di due parametri: la frequenza di precessione e la funzione di depolarizzazione. La prima contiene informazioni legate al tipo di interazione magnetica in quanto somma vari contributi, es. iperfine di contatto, dipolare, diamagnetico, etc. La comprensione piena di questo parametro è legata alla conoscenza della posizione dei siti reticolari in cui il muone si impianta, non sempre di facile individuazione. La misura della frequenza $\omega_{L}$ in funzione della temperatura fornisce informazioni utili alla determinazione della temperatura e dell'ordine della transizione magnetica e talvolta del modello di campo medio che descrive il materiale. La funzione di depolarizzazione $G(t)$ fornisce informazioni preziose, anche in sistemi non magneticamente ordinati, quali la natura statica o dinamica della distribuzione dei campi, la dinamica di spin, le lunghezze di correlazione del parametro d'ordine magnetico, la presenza di spin-density-wave, etc.

\section{VANTAGGI SPECIFICI DELLA SPETTROSCOPIA MUONICA}

La spettroscopia del muone è utilizzata principalmente per studi di base in fisica della materia condensata e chimica. In generale, si ottengono informazioni complementari a quelle fornite da altre tecniche sperimentali come il neutron scattering e le risonanze magnetiche nucleare o elettronica. Alcuni dei vantaggi specifici della spettroscopia del muone sono i seguenti:

1) è estremamente sensible a campi magnetici interni molto piccoli $(\sim 0.1$ Gauss $)$, sia di origine elettronica che nucleare.

2) È molto efficace anche in sistemi magnetici diluiti o con momenti disordinati (spin glass) o ordinati a corto raggio. Infatti il piccolo raggio di azione dell'interazione magnetica col singolo muone $(\sim 1$ nanometro), conferisce alla tecnica un potere di indagine microscopico. Questo favorisce anche la possibilità di dare informazioni quantitative sulla coesistenza/competizione di fasi diverse contemporaneamente presenti in un materiale (per esempio magnetismo e superconduttività).

3) L'intervallo di fluttuazioni magnetiche misurabile è compreso fra 10 
$\mathrm{kHz}$ e $1000 \mathrm{MHz}$, permettendo di colmare il gap tra il neutron scattering e la risonanza magnetica.

4) La tecnica può essere utilizzata in ogni materiale in quanto non è legata alla presenza di uno specifico suo componente, come nella risonanza magnetica o nella spettroscopia Mossbauer. Le condizioni di misura possono essere piuttosto varie, per esempio qualunque temperatura, campi magnetici fino a 8 Tesla, elevate pressioni, applicando campi a radiofrequenza etc.

5) Permette di misurare materiali di massa e dimensioni ridotti e in qualunque forma (monocristalli, policristalli o film). Attualmente la superficie esposta al fascio di muoni può essere ridotta fino a $0.1 \mathrm{~cm}^{2}$.

\section{RECENTI SVILUPPI}

Sebbene la possibilità di utilizzare i muoni per investigare le proprietà dei materiali fosse stata intuita già alla fine degli anni ‘ 50 , la spettroscopia del muone si è sviluppata solo attorno agli anni '80 e attualmente si può realizzare in quattro grandi centri di ricerca internazionali: in Canada, in Giappone e due in Europa (Fig. 6).

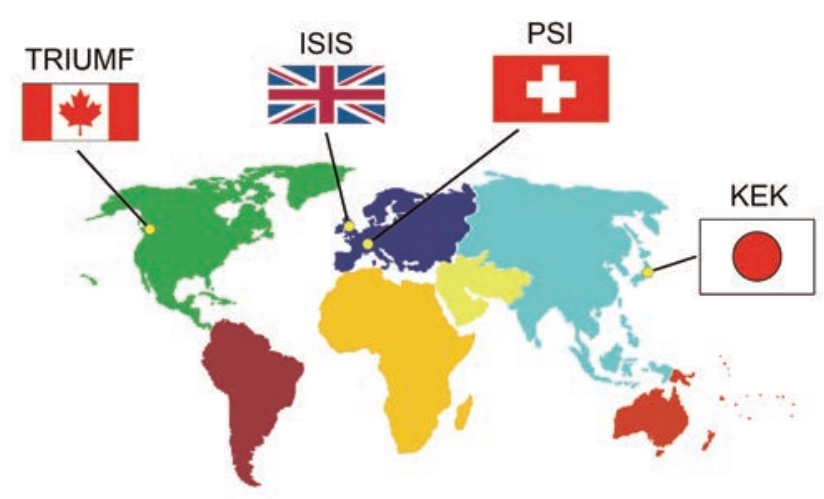

Fig. 6 - Al mondo sono quattro i Centri di Ricerca in cui è attualmente possibile realizzare esperimenti di spettroscopia muonica: TRIUMF a Vancouver (Canada), ISIS nelle vicinanze di Oxford, PSI nelle vicinanze di Zurigo, KEK a Tsukuba (Giappone).

I più recenti sviluppi riguardano la possibilità di utilizzare la tecnica in nuove condizioni sperimentali. Fra i progressi più significativi degli ultimi 10 anni vanno segnalati i tre seguenti: 
1) messa a punto e standardizzazione di uno spettrometro per la realizzazione di misure al variare della pressione esterna (fino a $30 \mathrm{kbar}$ ) presso il PSI in Svizzera. Questa tecnica richiede l'impiego di muoni che decadono da pioni in moto, in modo da sfruttare la loro energia cinetica per superare le robuste pareti del materiale della cella a pressione così da poter raggiungere il materiale in esso contenuto.

2) Ottimizzazione di un sistema di frenamento di muoni per lo studio di film sottili magnetici presso il PSI in Svizzera. Ad oggi è possibile controllare la profondità di penetrazione del muone con una precisione di una decina di nanometri, permettendo di selezionare "fette" differenti del materiale, essenziale per esempio nello studio di ossidi multistrato polifunzionali.

3) Realizzazione recentissima di uno spettrometro con possibilità di impiego di campi magnetici intensi (fino a 5 Tesla) presso ISIS nel Regno Unito. Un ulteriore spettrometro con un magnete fino a 10 Tesla è in corso di realizzazione presso il PSI. Questi strumenti offrono nuove opportunità per lo studio di fenomeni magnetici, elettrici e chimici in condizioni finora impossibili da ottenere in esperimenti di spettroscopia muonica. 


\section{RIFERIMENTI BIBLIOGRAFICI}

A. Yaouanc, P. Dalmas de Rèotier, Muon Spin Rotation, Relaxation and Resonance: Applications to Condensed Matter, Oxford University Press, Oxford (2011).

A. Schenck, Muon Spin Rotation Spectroscopy, Bristol: Adam Hilger (1985).

S.J. Blundell, Spin-polarized Muons in Condensed Matter Physics, Contemporary Physics 40, 175 (1999).

P. Dalmas de Rèotier and A. Yaouanc, Muon Spin Rotation and Relaxation in Magnetic Materials, J. Phys. Condens. Matter 9, 9113-9166 (1997).

P. Bakule, E. Morenzoni, Generation and Applicationn of Slow Polarized Muons, Contemporary Physics 45, 203-225 (2004).

Kanetada Nagamine, Introductory Muon Science, Cambridge University Press (2003).

Per un corso introduttivo su web si veda: http://www.fis.unipr.it/ derenzi/derenzi.html 